\title{
Questes
}

Revue pluridisciplinaire d'études médiévales

$25 \mid 2013$

L'Habit fait-il le moine?

\section{Une cour cousue d'or. Les ornements précieux utilisés par le duc Philippe le Bon}

\section{Élise Banjenec}

\section{OpenEdition}

\section{Journals}

Édition électronique

URL : http://journals.openedition.org/questes/124

DOI : 10.4000 /questes. 124

ISSN : 2109-9472

\section{Éditeur}

Les Amis de Questes

\section{Édition imprimée}

Date de publication : 15 avril 2013

Pagination : 45-64

ISSN : 2102-7188

\section{Référence électronique}

Élise Banjenec, « Une cour cousue d'or. Les ornements précieux utilisés par le duc Philippe le Bon», Questes [En ligne], 25 | 2013, mis en ligne le 01 janvier 2014, consulté le 19 avril 2019. URL : http:// journals.openedition.org/questes/124; DOI : 10.4000/questes.124 


\title{
Une cour cousue d'or. Les ornements précieux utilisés par le duc Philippe le Bon
}

\author{
Élise BANJENEC \\ Université Paris-Sorbonne (Paris IV)
}

«L'habit ne fait pas le moine». La mise en garde portée par le proverbe est sans équivoque: il ne faut pas se fier aux apparences. Le costume $^{1}$ est alors conçu comme un outil du paraître et de la démonstration sociale. Si l'habit du moine se doit de souligner l'humilité et le renoncement aux biens de ce monde pratiqués par son porteur, celui de la noblesse répond à l'adage inverse. L'ostentation est de rigueur pour signifier une condition sociale élevée. Quoi de mieux pour satisfaire à cet objectif qu'un bijou, une ceinture richement ouvragée ou des broderies de pierres précieuses? Pour nous, l'habit évoque avant tout un ensemble de pièces de tissu. Cependant, les éléments aujourd'hui désignés sous le nom d'«accessoires de mode» font partie intégrante du costume au Moyen Âge. Ils le magnifient, l'ornent, le complètent, et parfois même se substituent à lui. L'étude de la place tenue par l'orfèvrerie dans l'habit médiéval à travers un exemple prestigieux parait donc essentielle pour répondre à l'une des problématiques soulevées par le thème de ce bulletin : la question du statut ornemental du vêtement et de son utilisation comme moyen de représentation sociale.

Philippe le Bon, duc de Bourgogne (1419-1467), compte parmi les plus grands princes de l'Europe du $\mathrm{XV}^{\mathrm{e}}$ siècle. Ce duc, connu pour ses

${ }^{1}$ Dans cet article, le terme costume est entendu comme désignant l'ensemble des vêtements et ornements portés par un individu. Il n'a pas le sens restrictif de « costume théâtral » qu'on lui donne souvent aujourd'hui, mais est employé comme synonyme d'《habit». À l'époque de Philippe le Bon, l'« habit» inclut en effet, outre l'ensemble des vêtements qui couvrent le corps, la coiffure et la chaussure. Voir infra, Introduction. 
somptueuses fêtes, son luxe ostentatoire et ses nombreux dons, s'est bâti une image de seigneur parmi les plus riches et les plus puissants de cette époque, et ce en grande partie grâce aux objets précieux. Le nombre d'objets d'orfèvrerie médiévaux parvenus jusqu'à nous étant très réduit, les archives de la Recette générale de toutes les finances conservées à Lille ${ }^{2}$ et les chroniques du $\mathrm{XV}^{\mathrm{e}}$ siècle $\mathrm{e}^{3}$ fournissent de précieux renseignements sur la commande artistique du duc de Bourgogne. Le vocabulaire employé dans ces documents et les informations qu'ils apportent sur l'utilisation des parures précieuses soulignent l'appartenance de ces dernières au costume, et font apparaître la fonction politique et sociale qu'elles pouvaient revêtir. La façon dont ces atours étaient en effet perçus et employés pouvait différer selon le type de vêtement à orner, la charge ou le rang de son porteur et la circonstance dans laquelle il était porté.

\section{L'orfèvrerie et le costume : de la simple juxtaposition à la fusion}

$\mathrm{Au}$ Moyen Âge, quantité de bijoux pouvaient être ajoutés au costume, tels que les colliers, les bagues, ou encore les couronnes. D'autres objets, tels que les fermaux, les agrafes ou les ceintures, avaient plus particulièrement le rôle de lier entre eux plusieurs vêtements ou parties de vêtement. Enfin, les vêtements civils ou militaires pouvaient être brodés d'or, d'argent, de perles et de pierres précieuses. C'est par cette dernière catégorie de pièces d'orfèvrerie qui possède une relation particulière avec

\footnotetext{
${ }^{2}$ Les documents de la Recette générale de toutes les finances des ducs de Bourgogne mentionnés dans cet article sont conservés dans la série B des archives départementales du Nord à Lille.

${ }^{3}$ Mathieu D'Escouchy, Chroniques de Mathieu d'Escouchy, Gaston DE FreSNE DE Beaucourt (éd.), Paris, Renouard, «Publications pour la Société de l'Histoire de France », 1863-1864; OlIVIER DE LA MARCHE, Mémoires d'Olivier de La Marche: maître d'hôtel et capitaine des gardes de Charles le Téméraire, Henri BEAUNE et Jules D’arbaumont (éd.), Paris, Renouard, "Publications pour la Société de l'Histoire de France », 1883-1888; GeORges Chastellain, Euvres, Joseph-Bruno-MarieConstantin Kervyn De Lettenhove (éd.), Bruxelles, F. Heussner, « Euvres de Georges Chastellain », 1864.
} 
le costume, plus étroite et moins souvent évoquée, que la présente étude débutera. Certains manteaux, paletots ou chaperons étaient ainsi véritablement couverts d'orfèvrerie. De petites pièces d'or et d'argent, sous forme de «clinquants ${ }^{4}$ ou encore de «paillettes» pouvaient être appliquées «branlantes » c'est-à-dire pendantes, ou encore cousues sur le tissu pour ne montrer qu'une seule face ${ }^{5}$. Les brodeurs et les orfèvres étaient ainsi susceptibles de travailler ensemble à la confection de ces vêtements luxueux. Sous le principat de Philippe le Bon, les matériaux précieux étaient fournis soit par l'orfèvre, soit directement par le commanditaire comme nous le verrons plus loin.

Le vocabulaire employé dans les documents comptables bourguignons présente un intérêt particulier. Lorsqu'une pièce de tissu décorée d'orfèvrerie est décrite dans un article de comptes, le mot orné n'est en effet jamais employé : les termes utilisés sont plutôt mis, brodé ou encore garni. Si garnir peut effectivement signifier «orner», ce mot comporte toute une série d'autres sens. Il exprime d'abord une idée de protection ou de défense et, au sens figuré, de garantie. Il est particulièrement employé dans le domaine des arts militaires, où cette valeur est liée au fait d'être muni de l'équipement approprié : maintes significations de garnir tournent en effet autour des idées d'approvisionnement ou d'équipement. C'est à partir de ce dernier sens que garnir en arrive à signifier « orner » : dans cette dernière acception, garnir inclut l'action de «doubler » un vêtement, et peut suggérer l'abondance, puisque garni de peut aussi vouloir dire «être plein de», «être pourvu

\footnotetext{
${ }^{4}$ Le clinquant est un ornement brillant fait de lamelles d'or ou d'argent. Pour la signification des termes techniques voir Victor GAY, Glossaire archéologique $d u$ Moyen âge et de la Renaissance, Paris, Picard, 1883-1928, 2 vol., ainsi que le Trésor de la Langue Française Informatisé, http://atilf.atilf.fr/.

${ }^{5}$ Voir Sophie Jolivet, Pour soi vêtir honnêtement à la cour de monseigneur le duc de Bourgogne : costume et dispositif vestimentaire à la cour de Philippe le Bon de 1430 à 1455, thèse dactylographiée de doctorat sous la direction de Vincent TABBAGH, Université de Bourgogne, soutenue en 2003, p. 159.
} 
de $»^{6}$. Le champ sémantique de garnir réunit donc deux idées qui, pour nos esprits modernes, auraient pu paraître antithétiques: la protection et l'ornement, c'est-à-dire en quelque sorte l'utile et l'agréable. Ces deux réseaux de connotations aujourd'hui opposées semblent en effet s'appuyer tous deux sur le sème de l'abondance, l'idée d'être pourvu. Ces pièces d'orfèvrerie étaient donc considérées comme faisant partie intégrante du vêtement, le « remplissant » pour lui permettre de répondre, par le simple fait de le porter, à une fonction particulière d'ostentation au service du paraître et de la distinction sociale.

\section{Des uniformes précieux pour un entourage prestigieux}

Malgré le grand intérêt que portait Philippe le Bon aux parures précieuses, leur coût extrêmement élevé ne lui permettait pas d'en pourvoir tous les membres de sa cour. Parmi eux, les archers de corps ducaux font donc figure de privilégiés. Leur costume fait en effet l'objet d'une attention constante tout au long du principat de Philippe le Bon. Les archers, qui forment la garde rapprochée du prince dans tous ses déplacements et lors des cérémonies, constituent un groupe militaire dont la tenue uniformisée était particulièrement soignée : leurs vêtements d'apparat sont adaptés aux circonstances de chaque événement. La présence d'ornements d'orfèvrerie est la seule composante de leur costume à n'avoir jamais été supprimée, alors même que la diminution des moyens financiers ducaux la fait quasiment disparaître des habits du reste des gens de cour : "alors que la broderie d'orfèvrerie fut, passé le siège de Calais ${ }^{7}$, en nette perte de vitesse, les archers étaient la seule catégorie de personnel pour lesquels on a

\footnotetext{
${ }^{6}$ Voir Pierre CROMER, entrée « garnir », dans Robert MARTIN et Bernard COMBETTES (dir.), Dictionnaire du Moyen Français (1330-1500), ATILF, Nancy, 2010, http://www.atilf.fr/dmf.

${ }^{7}$ Philippe le Bon assiège la ville de Calais en 1436 dans le but de la reprendre à ses anciens alliés anglais. Après avoir été grandement malmenées, les troupes bourguignonnes doivent se retirer en Flandres.
} 
continué à réaliser des riches parures d'orfèvrerie, très coûteuses $)^{8}$. Ces archers se distinguent donc par le port de riches vêtements appelés «paletots». Ces habits du dessus doivent d'ailleurs leur nom, selon Michèle Beaulieu et Jeanne Baylé ${ }^{9}$, aux applications d'orfèvrerie dont ils ont été ornés avec constance. Ce vêtement avait un usage aussi bien militaire que d'apparat civil ${ }^{10}$.

Le renouvellement des paletots des archers a été très fréquent et semble avoir été motivé par des circonstances particulières: mariages, cérémonies de l'ordre de la Toison d'or, rencontres diplomatiques ou campagnes militaires. Ainsi, la tenue de sa garde personnelle est un élément suffisamment essentiel de l'ostentation ducale pour qu'à l'occasion du Banquet du Faisan Philippe le Bon emprunte à sa femme, Isabelle du Portugal,

cinquante marcs d'argent ${ }^{11}$ en vaisselle que elle lui presta pour de ce estre faites orfaverie et paillettes dont furent bordees certain grant nombre de robes, et icelui seigneur fist faire et donner a pluiseur nobles hommes et autres ses archiers de corps pour les porter le jour d'un banquet qu'il fist en sa ville de Lille ou mois de fevrier $1453^{12}$.

Ce banquet est en effet un événement d'envergure pour Philippe le Bon : il y officialise par un serment sa volonté de défendre la chrétienté en Turquie, en réaction à la prise de Constantinople en 1453. Ces festivités sont donc doublement importantes aux yeux du duc, puisqu'elles réactualisent le projet de croisade qu'il a toujours nourri. Le Banquet du Faisan représente alors pour lui l'occasion de mettre en œuvre toutes sortes de moyens afin d'apparaître aux yeux de toute l'Europe comme un grand seigneur mettant son immense richesse au service de la chrétienté. D'après ce que rapportent

\footnotetext{
${ }^{8}$ Sophie Jolivet, Pour soi vêtir honnêtement..., op. cit., p. 643.

${ }^{9}$ Michèle BeAulieu et Jeanne BAYlé, Le Costume en Bourgogne, Paris, PUF, 1956, p. $56-57$.

${ }^{10}$ Sophie Jolivet, Pour soi vêtir honnêtement..., op. cit., p. 115

${ }^{11}$ Soit environ 12,24 kg.

${ }^{12}$ Lille, Archives Départementales du Nord (ADN), B 2017, $\mathrm{f}^{\mathrm{o}} 206 \mathrm{r}^{\mathrm{o}}$ et v . $^{\mathrm{o}}$
} 
les chroniqueurs ${ }^{13}$, le duc y expose notamment de la vaisselle précieuse très coûteuse en quantité impressionnante ${ }^{14}$. Les dépenses extravagantes réalisées pour ce banquet l'ont ainsi contraint à emprunter la vaisselle de l'hôtel de la duchesse pour fournir l'argent du costume de ses archers. Or le chroniqueur Olivier de la Marche précise que ces derniers gardaient les portes d'entrée de la salle de réception: ils étaient donc les premiers membres du personnel que les convives voyaient à leur arrivée ${ }^{15}$, ce qui renforçait leur rôle essentiel dans l'ostentation de la cour ducale.

Le second trait marquant de cet uniforme réside dans le fait que l'orfèvrerie est employée différemment sur les paletots des archers, ceux de leurs capitaines, et ceux des capitaines qui sont aussi des chevaliers. Ainsi en 1455, le brodeur Thierry du Chastel est payé pour avoir brodé

sur les 4 paltos desdits 4 capitaines chacun quatre onces d'argent et, sur chacun des autres 300 , deux onces font lesdits 77 marcs $^{16}$ qui au pris de sept livres seize solz le marc valent $6001.12 \mathrm{~s}$. A lui pour avoir fait mettre iceulx $77 \mathrm{~m}$. d'argent en paillettes au pris de quarante huit solz le marc $1841.16 \mathrm{~s}$. A lui pour la dorreure des paillettes mises sur l'un des paltos desdits 4 capitaines pour ce qu'il est chevalier $41.16 \mathrm{~s}^{17}$

\footnotetext{
${ }^{13}$ Olivier de LA MARChe, Mémoires..., éd. cit., vol. II, p. 353 et 379 : « En celle salle, au plus près de la table, avoit ung hault buffet chargé de vaisselle d'or et d'argent et de potz de cristal garnys d'or et d'argent et de pierreries [...]. Tantost après fut apporté le vin et les espices, lesquelles espices estoient en sept dragoeuers, dont la pluspart estoient de pierreries »; MATHIEU D'EsCOUCHY, Chroniques..., éd., cit., vol. II, p. 137 : «En icelle salle, au plus prez de la table, avoit ung grant drechoir et hault, qui estoit tout chargié de vaisselle d'or et d'argent et d'autres comme pos, flacons, couppes, couvertes, aigières de cristal et de voirre de moult d'estranges et diverses manières et coulleurs garniz d'or et de riche pierre moult gentement ouvrez ».

${ }^{14}$ Cette vaisselle n'était d'ailleurs pas forcément la sienne : il avait dû en emprunter une partie, comme il l'avait déjà fait pour la fête de l'ordre de la Toison d'or de 1440. Voir Élise BANJENEC, «L'orfèvrerie comme outil politique : les commandes de Philippe le Bon, duc de Bourgogne, entre 1440 et 1450 », Actes de la journée doctorale en Histoire de l'Art «Art et Politique » du 31 mai 2011, Université Paris-Sorbonne, à paraître.

${ }^{15}$ Olivier de LA MARChe, Mémoires ..., éd. cit., vol. II, p. 349.

${ }^{16}$ Soit environ $18,85 \mathrm{~kg}$.

${ }^{17}$ Lille, ADN, B 2020, $\mathrm{f}^{\mathrm{o}} 460 \mathrm{v}^{\mathrm{o}}$ et $461 \mathrm{r}^{\mathrm{o}}$.
} 
Les capitaines portent une quantité d'argent deux fois plus importante que celle octroyée aux simples archers. Les paillettes de l'unique chevalier présent parmi les capitaines sont, de plus, dorées pour le distinguer comme appartenant à un rang plus élevé ${ }^{18}$. L'orfèvrerie brodée sur les uniformes marque donc sans appel la hiérarchie au sein du corps des archers.

Lors du fameux Banquet du Faisan, les archers de corps n'ont pas été les seuls à recevoir de somptueux vêtements de la part de Philippe le Bon. Le duc met en scène son vœu de croisade de manière très théâtrale. Avant la prestation du serment, une foule de personnages se succède devant les convives au cours des différents entremets. Lors du dernier de ces intermèdes, Grâce-Dieu, une figure féminine allégorique portant un costume religieux, vient devant le duc pour lui présenter douze demoiselles accompagnées par douze chevaliers: elles personnifient douze vertus indispensables à la réussite de la croisade - les trois vertus théologales, Foi, Espérance et Charité, les quatre vertus cardinales, Courage, Justice, Prudence et Tempérance, et cinq vertus chevaleresques, Vérité, Largesse, Diligence, Raison et Vaillance. Ces figurants se présentent dans de fabuleux atours décrits par Olivier de la Marche :

Et après vindrent douze chevaliers, chascun menant une dame par la main, et estoient habillez de pourpoinctz cramoisys et de palletocz à manches, la moitié gris et l'aultre noir, de satin brodé de feuillage et chargé d'orfavrerie; et avoient chapeaux de velours noir, orfavrerisés comme lesdits palletocz [...]. Et lesdictes douze dames furent vestues de cottes simples de satin cramoisy, bordées de letices ${ }^{19}$; et par-dessus avoient en maniere d'une chemise de si fine toille, qu'on vit la cotte parmy; et

\footnotetext{
${ }^{18}$ On trouve d'autres exemples de ce type : Lille, ADN, B $1972 \mathrm{f}^{\mathrm{o}} .237 \mathrm{v}^{\mathrm{o}}$ et $238 \mathrm{r}^{\circ}$ (en 1441 ) ; Lille, ADN, B 1975, $\mathrm{f}^{\circ} 175 \mathrm{v}^{\circ}$ et $176 \mathrm{r}^{\circ}$ (en 1443) ; Lille, ADN, B 1982, $\mathrm{f}^{\circ} 236 \mathrm{v}^{\circ}$ (en 1445); Lille, $\mathrm{ADN}, \mathrm{B} 1988, \mathrm{f}^{\circ} 242 \mathrm{v}^{\circ}$ (fête de l'ordre de la Toison d'or à Gand en 1445 ) ; Lille, ADN, B 2004, $\mathrm{f}^{0} 358 \mathrm{r}^{\circ}$ et $358 \mathrm{v}^{\circ}$ (en 1450); Lille, ADN, B 2012, $\mathrm{f}^{0} 323 \mathrm{v}^{\circ}$ (en 1453) ; Lille, ADN, B 2030, $\mathrm{f}^{0} 363 \mathrm{r}^{\circ}, 363 \mathrm{v}^{\circ}, 364 \mathrm{r}^{\circ}$ et $364 \mathrm{v}^{\circ}$ (préparation du voyage en Turquie en 1458).

${ }^{19}$ La létice est la fourrure de couleur blanche de la belette des neiges.
} 
avoient ung atour ${ }^{20}$ tout rond à la façon de Portugal, dont les bourreletz $^{21}$ estoient à maniere de rauces ${ }^{22}$; et passoient par derriere, ainsi que pattes de chapperons pour hommes, de deliés voletz ${ }^{23}$ chargez d'orfavrerie d'or branlant; et furent leurs visaiges couverts du volet ${ }^{24}$.

Le chroniqueur Mathieu d'Escouchy ajoute même à cette description que les paletots des chevaliers étaient «brodez de feullages d'or et chargiéz d'orfaverie très richement», que leurs coiffes étaient «bordez et orphaverisiés comme lesdis palletos» et que les dames portaient des « déliés voiles, chargiez et brodez, et pareillement à bourlès desdis atours d'orfaverie d'or branllant et esmaillé moult gentement. Et avoient au col colers d'or aournez de pierries touttes pareilles $»^{25}$. Les archives ducales ne font pas mention des costumes des dames. Cependant, les habits des chevaliers y sont bien mentionnés puisqu'un certain Jean de Dessetinghen est rémunéré «pour la façon de 12 paltos de satin noir et gris chargiés d'orfaverie que il a semblablement fait et donner a douze gentilz hommes de son hostel le jour de sondit banquet $»^{26}$. Une différence se fait jour entre les uniformes d'apparat des archers ducaux et ces déguisements transformant les chevaliers et leurs dames en vertus. Si les broderies et autres incrustations d'orfèvrerie se limitent, pour les archers, à leur paletot, les personnifications du divertissement les arborent sur leur paletot mais aussi sur leur coiffe. Ce développement des décorations précieuses transforme alors ces éminents membres de la cour ducale en de

\footnotetext{
${ }^{20}$ L'atour désigne ici un bonnet ajouré, chargé d'orfèvrerie, selon la mode portugaise du milieu du $\mathrm{XV}^{\mathrm{e}}$ siècle. Voir Michèle BEAUlieu et Jeanne BAYLÉ, Le Costume en Bourgogne..., op. cit., p. 89.

${ }^{21}$ Le bourrelet est une couronne faite de bourre, diversement agrémentée, servant de base à une coiffure de femme ou à un chaperon d'homme. Le chaperon, quant à lui, est une sorte de bonnet enveloppant la tête prolongé par un pan d'étoffe dont on se sert comme d'une écharpe.

22 « Roses ».

${ }^{23}$ Le volet est une pièce d'étoffe flottant au vent.

${ }^{24}$ Olivier de La MARche, Mémoires..., éd. cit., vol. II, p. 372.

${ }^{25}$ Mathieu D'Escouchy, Chroniques..., éd. cit., t. II, p. 226-228.

${ }^{26}$ Lille, ADN, B 2017, $\mathrm{f}^{\mathrm{o}} 306 \mathrm{v}^{\mathrm{o}}$ et $307 \mathrm{r}^{\circ}$.
} 
somptueuses allégories des vertus chrétiennes et chevaleresques, personnages dont la beauté évoque l'éclat du rayonnement divin ${ }^{27}$. Ainsi, par la richesse et la splendeur de ses ornements, le costume des gens de la cour ducale tient une place de première importance dans les différentes formes de mise en scène du pouvoir ducal, qu'elles soient d'ordre cérémoniel ou véritablement théâtral. Ces vêtements sont alors tout aussi remarqués par les convives que les buffets remplis de vaisselle précieuse.

\section{Les parures ducales : un outil politique de premier ordre}

Si le duc porte une attention particulière au costume de son entourage, il est encore plus attentif à sa propre toilette, qui ne laisse aucune place au hasard. Parmi les nombreuses parures que Philippe le Bon achète pour son propre usage, signalons tout d'abord les objets désignés dans les comptes sous le nom d'« écharpe ducale». Ils consistent en une bande de tissu mêlée de fils d'or et garnie de pierres précieuses et de perles, portée en écharpe, c'est-à-dire en biais par-dessus les vêtements, dans le cadre d'une cérémonie. L'une de ces écharpes apparaît dans les comptes ducaux dès 1436, année où l'orfèvre brugeois Jean Peutin est prié de préparer un diamant destiné à être brodé sur celle-ci : «a lui pour avoir mis en ung $\mathrm{clou}^{28}$ l'un des gros diamens de mondit seigneur nommé le diament

\footnotetext{
${ }^{27}$ L'idée sous-jacente est que la beauté sensible, et en particulier la luminosité des pierreries, peut évoquer l'éclat du rayonnement divin. Ce principe néoplatonicien est développé par le Pseudo-Denys l'Aréopagite vers l'an 500. Il est ensuite repris et diffusé par l'abbé Suger de Saint-Denis dans la première moitié du XII ${ }^{\mathrm{e}}$ siècle. Selon cette conception, le déploiement des ornements répond à une sorte de "métaphysique de la lumière », pour reprendre l'idée exprimée par Erwin Panofsky (Erwin PANOFSKY, Architecture gothique et pensée scolastique, précédé de L'abbé Suger de Saint-Denis, Pierre BourdiEU (trad. et postface), Paris, Éditions de Minuit, «Le sens commun», 1970, p.38-40; traduction de Gothic Architecture and Scholasticism, Cleveland/New York, The World Publishing Company, " Meridian Books », 1963).

${ }^{28}$ Le clou désigne ici un clou d'ornement fixé sur un vêtement qu'il sert à fermer.
} 
de Brabant pour icellui mettre en sa belle escherpe $91.12 \mathrm{~s} .{ }^{29}$. Puis, en 1443, le duc paie Jean Peutin pour avoir « assiz et mis en oeuvre plusieurs balaiz $^{30}$ en la belle escharpe de mondit seigneur $181 .{ }^{31}$. Philippe le Bon utilise en effet cette même année sa «belle écharpe » lors d'un dîner organisé à Besançon pour traiter avec le roi des Romains Frédéric de Habsbourg, qui conteste alors les possessions du duc de Bourgogne en Hainaut, Hollande, Zélande et Brabant ${ }^{32}$. Olivier de la Marche décrit dans ses Mémoires la forte impression produite par cet objet particulièrement somptueux - ce sentiment est d'ailleurs confirmé par l'estimation exagérée de son prix :

Chascun jour visitoit le duc de Bourgoingne le Roy et le dimenche suyvant, fit le duc ung grand et riche disner où le Roy et les seigneurs de sa compaignie disnerent, et ay bien souvenance que le duc porta celluy jour une echarpe d'or garnye de balais et de perles, que l'on extimoit valoir plus de cent mille escuz ${ }^{33}$.

Philippe le Bon choisit ainsi d'emporter son écharpe en déplacement et de la porter en présence de Frédéric IV afin de s'imposer à lui comme un prince riche et puissant, ce qui devait peser dans le cadre des pourparlers. Les objets d'orfèvrerie sont alors utilisés pour leur pouvoir de valorisation et de fascination.

En 1445, le brodeur Thierry du Chastel travaille également à la réalisation d'une écharpe, qui ne semble certes pas être exactement la même que celle citée précédemment au regard de la nature et du nombre de pierres utilisées, mais dont la description donne une idée un peu plus

\footnotetext{
${ }^{29}$ Sophie Jolivet, Pour soi vêtir honnêtement..., op. cit., p. 122 et Lille, ADN, B 1957, $\mathrm{f}^{\mathrm{o}} 376 \mathrm{v}^{\mathrm{o}}$.

${ }^{30}$ Le rubis balais, abrégé ici en «balais », désigne un rubis d'un rouge lavé, cendré ou virant au rose originaire du Badakhchan, une province montagneuse de l'extrême Nord-Est de l'Afghanistan.

${ }^{31}$ Sophie Jolivet, Pour soi vêtir honnêtement..., op. cit., p. 122 et Lille, ADN, B 1978, $\mathrm{f}^{\mathrm{o}} 266 \mathrm{v}^{\mathrm{o}}$.

${ }^{32}$ Voir Bertrand Schnerb, L'État bourguignon, Paris, Perrin, « Tempus », 1999, p. 218.

${ }^{33}$ Olivier de La Marche, Mémoires..., éd. cit., vol. I, p. 278-279.
} 
précise de ce type d'objet réservé au duc ${ }^{34}$. Elle est en effet brodée de motifs représentant la croix de Saint André, des fusils ${ }^{35}$, des flammes et la devise ducale ${ }^{36}$ - tous ces éléments renvoyant aux emblèmes de Philippe le Bon et de son ordre de la Toison d'or. Elle est également frangée sur tous les bords et porte cinq gros rubis balais et quatre diamants pointus enchâssés dans de 1'or ${ }^{37}$. Puis, en 1447, l'orfèvre bruxellois Étienne de la Poulle refait dix-sept chatons d'or et une brochette ${ }^{38}$ pour fermer l'écharpe du duc. Il s'agit apparemment d'une troisième écharpe, puisqu'il assied dans les nouveaux et les anciens chatons trente-trois rubis balais et place une feuille d'or sous chacun d'eux afin qu'ils brillent davantage ${ }^{39}$. L'absence de mention du fameux «diamant de Brabant » dans ces derniers articles indique qu'il s'agit vraisemblablement d'écharpes différentes de celle citée par Olivier de la Marche. Ce n'est pas du tout improbable, puisque le duc Jean sans Peur, père de Philippe le Bon, a lui-même possédé quatre écharpes si l'on se réfère à l'inventaire de ses joyaux dressé en $1420^{40}$. La volonté de Philippe le Bon de garnir l'une de ses écharpes d'une pierre apparemment remarquable telle que le «diamant de Brabant» ou encore l'envie d'en faire améliorer une autre en y ajoutant des feuilles d'or

\footnotetext{
${ }^{34}$ Voir Sophie Jolivet, Pour soi vêtir honnêtement..., op. cit., p. 122.

${ }^{35}$ Le fusil [italique] est une petite pièce d'acier en forme de B que l'on frottait contre un silex pour en faire jaillir des étincelles. Le fusil et la pierre à feu constituent les deux éléments de la devise de Philippe le Bon.

${ }^{36}$ Rappelons que la devise désigne une figure emblématique accompagnée d'une courte formule qui, généralement, s'y rapporte. Elle allie donc écriture et représentation figurée.

${ }^{37}$ Lille, ADN, B 1982, $\mathrm{f}^{\mathrm{o}} 237 \mathrm{v}^{\circ}$ : « pour la facon dont brode bien richement de pierrerye et orfaverye a croix de saint andrieu, fuzilz, flambes, et autres devises de mondit seigneur sur une bende pour lui a porter en escharpe $141.8 \mathrm{~s}$. A lui pour sept aulnes de franges pour frangier ladite bende tout alentour au pris de six solz chacune aulne valent $42 \mathrm{~s}$. A lui pour ung marc cinq onces et dix huit esterlins d'or pour mettre et encasser cinq gros balais et quatre deamans pointus en or pour asseoir sur ladite bende et escharpe au pris de neuf solz $4 \mathrm{~d}$. chacun esterlins valent 139 1. A lui pour la facon d'avoir mis et assis lesdits balais et deamans oudit or sur ladite escharpe $321.8 \mathrm{~s}$. ».

${ }^{38}$ C'est-à-dire une petite broche.

${ }^{39}$ Lille, ADN, B 1997/60120.

${ }^{40}$ Voir Sophie Jolivet, Pour soi vêtir honnêtement..., op. cit., p. 122.
} 
montrent que ces écharpes devaient particulièrement satisfaire son désir d'ostentation. Cette observation est d'ailleurs confirmée par le témoignage d'Olivier de la Marche cité ci-dessus.

Les joutes qui ont précédé le Banquet du Faisan ont également été l'occasion pour Philippe le Bon d'éblouir ses convives par ses propres costumes. Le duc et deux de ses fils assistent en effet au tournoi, parés avec éclat. Philippe le Bon porte un collier d'or enrichi de diamants, de balais et de perles ainsi qu'un chaperon dont la cornette est elle aussi ornée de pierreries - une tenue qu'Olivier de la Marche juge digne d'un «prince puissant » :

Au jour de ce bancquet doncques, monseigneur Adolf, qui s'estoit fait crier le chevalier au Cigne, vint après disner, de très bonne heure, sur les rens, et fut accompaigné, du lieu où il estoit armé, par mondit seigneur le duc, par monseigneur de Charrolois, par monseigneur le bastard de Bourgoingne, vestuz tous trois de robes de velours sur velours noir; et avoient chascun ung collier d'or moult enrichiz de pierreries, comme diamans, balais et parles; et portoit mondit seigneur une cornettes ${ }^{41}$ à son chapperon, si riche de pierrerie que je ne le sçay aultrement extimer, fors habillement de prince puissant ${ }^{42}$.

En 1454, Thierry du Chastel est d'ailleurs payé «pour la brodure par lui faite sur la cornette de l'un des chapperons de mon avantdit seigneur sur laquelle ont pluiseurs gros perles et chieres pierieries 241 . $\rangle^{43}$. Ce couvrechef, qui semble correspondre à celui décrit par Olivier de la Marche, a permis à Philippe le Bon d'éblouir ses convives par son goût et sa richesse dès le commencement des festivités de 1454 .

Lors de l'entrée du nouveau roi Louis XI à Paris en 1461, la mise de Philippe le Bon, ainsi d'ailleurs que la profusion de richesses déployées par

\footnotetext{
${ }^{41}$ La cornette est la pointe du chaperon qui retombait en arrière ou s'enroulait sur la tête ou autour du cou. Elle a ensuite pris la forme d'une simple bande.

${ }^{42}$ Olivier de La MARche, Mémoires..., éd. cit., vol. II, p. 345.

${ }^{43}$ Lille, ADN, B 2017, f $\mathrm{f}^{\mathrm{3}} 307 \mathrm{r}^{\circ}$.
} 
sa suite, suscitent à nouveau beaucoup d'admiration auprès des spectateurs.

Georges Chastellain décrit le défilé en ces termes :

Sy vint l'heure que le duc de Bourgongne voulut partir de la ville pour aller au devant du roy et pour luy faire honneur à son entrée, de luy et de ses nobles vassaux. Et en effet, se disposant à ce, monta en son hostel d'Artois; et là se fist l'assemblée de tous ceux qui le devoient accompagner, [...] en tel nombre que l'un parmi l'autre, princes et barons se trouvèrent là ensemble deux cents quarante-trois chevaux couverts de drap d'or, d'orfèvrerie et de velours et de brodure, et les moindres de damas et de satins, dont le nombre estoit petit, sans encore les richesses et les habillemens de leurs corps et de leurs pages, dont oncques en ce royaume françois n'avoit esté vu, ne ouy cas pareil, ne qui y prist comparaison. Et en ceste estat, sans faire autre narration de leur joliveté, le duc party de son hostel et dressa son chemin vers la porte Saint-Denis, parmy celle grand rue, là où le peuple avoit tant haut et bas, et des dames aux fenestres que le nombre en fut inestimable, et s'esblouissoient les yeux des dames et des regardans sur les richesses et beauté de ceste compagnie. Et tant en furent esmerveilliés et espris de joye, que à peine les bouches ne leur souffroient à vuydier par paroles ce que les yeux leur faisoient comprendre superflues et d'admiration dignes.

Le duc de Bourgongne portoit une plume à son chapeau, de pris non extimable; et estoit garnie ycelle de neuf gros balais, cincq gros diamans, trois des plus grosses perles de la terre et claires à l'advenant, de soixante-deux autres perles de sorte et de grand pris; et au chanfrain de son cheval y avoit pareillement neuf gros balais autres, entresemés de perles sans nombre. Et en la salade ${ }^{44}$ portée après, estoit assis un riche balais de Flandres, l'outrepas ${ }^{45}$ de chrestienneté. Et, ce dit-on, les autres montures qui le sieuvoyent, avecques les couvertures d'orfèvreries, ne sont à réciter en fait de richesse et de grand pris, car les bouches n'y suffiroient à le mettre en compte, et les oyans

\footnotetext{
${ }^{44}$ La salade est une sorte de casque ouvert ou fermé, très bombé, à visière courte et à couvre-nuque.

${ }^{45}$ C'est-à-dire la « merveille », d'après l'entrée « outrepas » du DMF (Robert MARTIN et Bernard CombetTes (dir.), Dictionnaire du Moyen Français (1330-1500), ATILF, Nancy, 2010, http://www.atilf.fr/dmf).
} 
restiveroient en la créance, qui ne l'auroient vu. Par quoy sous ceste générale manière de parler, je couvre et comprens la pompe et richesse de tous les autres qui en ensieuvant le chef d'eux tous, leur duc, estoit haute et grande par extrême ${ }^{46}$.

Les nombreux procédés utilisés par le chroniqueur pour exprimer son émerveillement devant la splendeur du cortège ducal laissent imaginer l'effet produit par Philippe le Bon sur le peuple parisien pourtant «réputé pour être difficilement impressionnable $»^{47}$. Il faut cependant garder à l'esprit que, Georges Chastellain étant au service du duc, il ne manque certainement pas d'amplifier les réactions des parisiens. Cet événement tenait extrêmement à cœur à Philippe le Bon. La mort de Charles VII, qui n'avait cessé d'être hostile à la puissance bourguignonne, semblait marquer la fin des tensions entre celle-ci et la France. Le duc pensait alors que Louis XI serait mieux disposé à son égard, ne serait-ce que parce qu'en 1456 le dauphin, en révolte ouverte contre son père Charles VII, avait trouvé refuge à la cour de Bourgogne. Philippe le Bon espérait donc jouer un rôle politique de premier plan dans le gouvernement royal, ce qui explique son entrée triomphale à Paris ${ }^{48}$.

D'après ce récit, un ornement du costume ducal éclipse tous les autres: la plume, ou plumas, disposée sur son chapeau et ornée d'une grande quantité de pierres précieuses: «neuf gros balais, cincq gros diamans, trois des plus grosses perles de la terre et claires à l'advenant, de soixante-deux autres perles de sorte et de grand pris $»{ }^{49}$. Si les chapeaux pouvaient être recouverts d'ornements précieux, la seule plume qui en décorait parfois la cime suffisait donc à éblouir la foule. Les archives ducales complètent cette description en indiquant que Guillaume Piart,

\footnotetext{
${ }^{46}$ Georges Chastellain, Euvres..., éd. cit., vol. IV, p. 76-78.

${ }^{47}$ Bertrand SCHNERB, L'État bourguignon..., op. cit., p. 407.

${ }^{48}$ Il s'avèrera, par la suite, qu'il se fourvoyait puisque Louis XI poursuivra la politique anti-bourguignonne de son père.

${ }^{49}$ Georges Chastellain, Euvres..., éd. cit., vol. IV, p. 77.
} 
plumetier du duc, a été payé pour un «plumatz garny d'orfaverie branlant et paillecté de plusieurs paillectes d'argent doré qu'il a pieca fait et livré pour mondit seigneur en sa ville de Bruxelles » pour servir à son entrée à Paris $^{50}$. Les plumes coiffaient également souvent les casques des cavaliers de l'armée ducale. L'armure jouait en effet aussi un rôle important dans la politique d'ostentation de Philippe le Bon.

\section{La guerre par l'ornement : le cas des armures}

Malgré les pourparlers, le duc en arrivait parfois à utiliser les armes pour défendre ses intérêts. Dans le costume militaire, l'orfèvrerie intervient dans une démarche d'intimidation plus nette encore. Il était des armures aussi riches en orfèvrerie que certains somptueux habits civils. Olivier de la Marche évoque, dans ses Mémoires, la brillante armée de Philippe le Bon lorsque, après l'échec de la voie diplomatique à Besançon, l'armée ducale part de Dijon en 1443 vers le Luxembourg pour asseoir le pouvoir bourguignon dans ce duché ${ }^{51}$ :

Ses paiges estoient richement en point, et portoient divers harnois de teste garniz et ajolivez de parles, de diamans et de balais, à merveilles richement, dont une seule salades estoit extimée valoir cent mille escus d'or. Le duc de sa personne estoit armé gentement de son corps et richement ès gardes, tant de ses bras, comme de son harnois de jambes, dont icelles gardes, et le chanfrain de son cheval estoient tous pleins et enrichiz de grosses pierreries qui valoient un merveilleux avoir. ${ }^{52}$

Même si le chroniqueur estime à nouveau exagérément le prix des casques, sa description des vêtements et harnachements des hommes et chevaux est tout à fait crédible. Outre les vêtements d'apparat militaire des archers déjà

\footnotetext{
${ }^{50}$ Lille, ADN, B $2040 \mathrm{f}^{\mathrm{o}} 278 \mathrm{v}^{\mathrm{o}}$ et $279 \mathrm{r}^{\mathrm{o}}$.

${ }^{51}$ Celui-ci revenait en effet au duc de Bourgogne par le traité de Hesdin qu'il avait signé en 1441 avec la duchesse du Luxembourg. Cependant plusieurs partis s'en disputaient encore les droits.

52 Olivier de LA MARChe, Mémoires..., éd. cit., vol. II, p. 11-12.
} 
étudiés dans cet article, les armures faisaient également l'objet d'une attention particulière. Dans sa description de l'entrée de Louis XI à Paris en 1461 citée ci-dessus, Georges Chastellain, signale la «salade» du duc, portée à sa suite, sur laquelle était enchâssé « l'outrepas de chrestienneté », soit l'« un des plus beaux rubis qui fut au monde»d'après Charles Buttin $^{53}$. Plusieurs salades de cette richesse sont décrites dans les archives ducales : en 1466 par exemple, Philippe le Bon charge son garde des joyaux, Jacques de Brégilles, de se rendre à Bruges pour « faire garnir d'or et de pierres precieuses une sallade que mondit seigneur luy avoit ordonné faire faire pour porter ou voiage qu'il fist a la conqueste de Dinant $\gg{ }^{54}$.

Aucun casque de cette richesse n'est malheureusement parvenu jusqu'à nous. Cependant, une miniature du livre des Statuts et privilèges de Gand et de Flandre conservé à Vienne, qui représente «Les Gantois f[aisa]nt amende honorable à Philippe le Bon ${ }^{55}$, offre un témoignage visuel de la richesse de ces panoplies militaires ${ }^{56}$. Au premier plan, Philippe le Bon, monté sur un cheval blanc richement paré, fait face au peuple de Gand agenouillé. Le Maître des Privilèges de Gand et de Flandres a choisi un point de vue assez bas, donnant au duc un rôle dominant dans la composition. Sa volonté n'était pas de reproduire un

${ }^{53}$ Charles Buttin, Le Guet de Genève au XV siècle et l'armement de ses gardes, Genève/Anneçy, Kïnidig/J. Abry, 1910, p. 86.

${ }^{54}$ Lille, ADN, B 2061, $\mathrm{f}^{\mathrm{o}} 133 \mathrm{r}^{\circ}$ et $\mathrm{v}^{\mathrm{o}}$. Philippe le Bon procéda en effet au sac de Dinant en août 1466.

55 «Les Gantois font amende honorable à Philippe le Bon», Statuts et privilèges de Gand et de Flandre, Vienne, Österreichische Nationalbibliothek (ÖNB), 2583, fo $349 \mathrm{v}^{\circ}$, 1453. Voir supra, Cahier des illustrations, Fig. 1, p. 125.

56 Statuts et privilèges de Gand et de Flandre, Vienne, Österreichische Nationalbibliothek (ÖNB), 2583, $\mathrm{f}^{\mathrm{o}} 349 \mathrm{v}^{\circ}$. Ce manuscrit a été réalisé aux environs de 1453 pour Philippe le Bon. La scène dont il est question représente l'Amende honorable des citoyens de Gand à Ledeberg, dans les faubourgs de la cité, le 30 juillet 1453, quelques jours après la victoire du duc de Bourgogne sur la milice de Gand à Gavere. 
événement réel mais d'illustrer l'idée de « domination terrestre $»^{57}$, ce que Philippe le Bon devait apprécier particulièrement. Le duc porte une armure dont le précieux casque est remarquable. Cette salade semble être composée d'un casque par-dessus lequel est posé un tissu orné de fil d'or et de pierres précieuses qui le dissimule presque entièrement. Le cimier, ou la crête du casque, qui seul dépasse de ce turban d'étoffe est de plus orné d'un briquet bourguignon doré ${ }^{58}$.

Un casque semblable surmonté d'un turban est signalé dans un document plus tardif, datant d'août 1471, qui stipule le paiement effectif de Gérard Loyet, orfèvre de Charles le Téméraire, fils de Philippe le Bon, pour avoir estoffé ung chappeau d'achier et y faut a l'entour ung chappeau de duc d'or, garni de dix huit gros balais et de quarante bien grosses perles, et a l'entour desdiz balais de grosses feuilles eslevees et percees a jour, et de plusieurs autres garnitures y servans, a l'entour desdites perles semblables fueilles, CV livres ${ }^{59}$.

Le décor d'orfèvrerie d'un casque pouvait ainsi se concentrer sur un chapeau venant prendre ensuite place sur la salade ou encore orner directement le fer du casque. Sur la même miniature, les cavaliers placés derrière le duc portent d'ailleurs des casques ornés à leur base de clous ou de boulons dorés ${ }^{60}$ directement œuvrés sur leur salade.

Même si cette miniature ne représente pas un casque ayant véritablement appartenu au duc, la mise en évidence de la somptuosité de son armure correspond tout à fait au but recherché par Philippe le Bon en arborant un tel costume : affirmer sa puissance et son autorité. La richesse

\footnotetext{
${ }^{57}$ Gregory Clark, dans Bernard Bousmanne et Thierry Delcourt (dir.), Miniatures flamandes : 1404-1482, catalogue d'exposition, Bruxelles/Paris, Bibliothèque royale de Belgique/Bibliothèque nationale de France, 2011, p. 182-185.

${ }^{58}$ Le briquet est le terme médiéval désignant le fusil.

${ }^{59}$ Hugo VAn DER Velden, The Donor's Image. Gerard Loyet and the Votive Portraits of Charles the Bold, Turnhout, Brepols, «Burgundica », 2000, p. 310-311 ; Lille, ADN, B 2089.

${ }^{60}$ Le boulon est une bossette de métal qui sert d'ornement.
} 
de son armée avait donc valeur d'intimidation de l'adversaire. Ce seigneur si riche et si puissant impressionnait sans nul doute l'armée adverse par ce biais et gagnait ainsi une partie de la guerre psychologique.

\section{Lorsque le joyau remplace le costume : tout un symbole}

Les éléments du costume précédemment étudiés sont intéressants car ils allient tissu ou armure et matériaux d'orfèvrerie. Ces derniers font ainsi partie intégrante du vêtement pour le magnifier. Cependant, les bijoux peuvent également, non plus s'unir au vêtement, mais se substituer symboliquement à lui. C'est le cas d'un joyau comptant parmi les objets les plus importants du principat de Philippe le Bon : le collier de l'ordre de la Toison d'or. Le duc crée l'ordre de chevalerie de la Toison d'or en 1430, dans le but d'affirmer son indépendance politique vis-à-vis de la France et de l'Angleterre mais aussi de partir en croisade, vœu qui ne sera pourtant jamais exaucé ${ }^{61}$. Cet ordre était de plus un excellent outil diplomatique permettant au duc de se concilier les grands personnages de Flandres et d'Europe. L'objet scellant cette entrée dans l'ordre était un collier dont le port était réglementé et obligatoire, contrairement au costume complet (manteau et chaperon écarlates) qui n'était porté que lors des cérémonies de l'ordre. Le collier était donc l'objet le plus symbolique de ce costume et se substituait à l'ensemble de l'habit pour désigner un chevalier de l'ordre.

$\mathrm{Au}$ chapitre de l'ordre qui se déroule à Gand en 1445, Philippe le Bon fait élire le roi Alphonse V d'Aragon comme chevalier de la Toison $d^{\prime}$ or $^{62}$. Cette alliance revêtait une importance particulière pour la réalisation de la croisade voulue par le duc. En effet, le roi d'Aragon possédait une puissante flotte, indispensable pour aller guerroyer contre les « mécréans »,

\footnotetext{
${ }^{61}$ Voir Bertrand SchNERB, L'État bourguignon ..., op. cit., p. 299-300.

62 Voir Françoise DE GRUBEN, Les Chapitres de la Toison d'or à l'époque bourguignonne (1430-1477), Louvain, Leuven University Press, « Mediaevalia Lovanensia », 1997, p. 242.
} 
et qui faisait défaut à Philippe le Bon. Le duc envoie donc un collier de l'ordre en Aragon et reçoit en retour celui de l'ordre de l'Étole et Jarre ${ }^{63}$. Cependant Alphonse V refuse le port quotidien du collier de la Toison d'or, qui le lie de façon trop importante au duc de Bourgogne. Il demande d'ailleurs explicitement à pouvoir évaluer les conflits avant d'y soutenir Philippe le Bon. Il veut ainsi séparer le domaine politique de l'engagement chevaleresque $^{64}$. Cette vision est assez différente de celle de Philippe le Bon. Ce dernier accepte tout de même la requête mais exige en retour les mêmes privilèges pour lui-même à l'égard de l'Étole et Jarre. Cet épisode montre que le port du collier, se substituant à celui du costume entier, désigne explicitement son porteur comme chevalier de l'ordre de la Toison d'or, donc comme un personnage socialement très important, mais surtout comme un allié politique du duc de Bourgogne.

Ces quelques exemples d'ornements précieux portés à la cour de Philippe le Bon montrent la diversité et la richesse de l'utilisation de l'orfèvrerie dans le costume, que ce soit dans sa forme ou dans sa fonction. Des uniformes militaires aux costumes de cérémonie, les gens de la cour ducale se devaient d'être vêtus le plus richement possible pour participer à l'image d'opulence et de puissance que le duc voulait donner à l'Europe entière. Au-delà de la simple volonté d'ostentation, on utilise ces précieux matériaux pour des raisons et dans des cadres assez variés. La quantité et la

\footnotetext{
${ }^{63}$ L'ordre de l'Étole et Jarre fut fondé par le père d'Alphonse V d'Aragon ; Lille, ADN, B 1992/59845 : « Jehan Peutin, orfevre demourant a Bruges, a fait pour mondit seigneur et par le commandement d'icelluy seigneur a moy delivré 2 coliers d'or dudit ordre de Thoison d'or [...] desquelz 2 coliers mondit seigneur a prins et retenu l'ung devers lui et l'autre a fait par moy baillier a Messir Guillebert de Lannoy, seigneur de Avillerual, son conseillier, chambellan et chevalier dudit ordre pour de par mondit seigneur le porter et presenter au roy d'Arragon auquel icelluy seigneur l'a envoié "».

${ }^{64}$ Voir Françoise DE GRUBEN, Les Chapitres de la Toison d'or..., op. cit., p. 246. Voir également Élise BANJENEC, « L'orfèvrerie comme outil politique... », art. cit.
} 
qualité des pièces de métal cousues sur les uniformes des archers servaient également à maintenir la hiérarchie. Les joyaux portés par le duc pouvaient quant à eux servir d'instrument de valorisation dans le cadre de pourparlers diplomatiques. Enfin, les armures richement décorées poursuivaient cette entreprise sur le champ de bataille. Comme son inventaire le laisse penser, Jean sans Peur possédait déjà tous ces types d'objets précieux et s'en servait très volontiers, perpétuant ainsi l'émulation artistique engagée par les princes des fleurs de lys ${ }^{65}$ dans la seconde moitié du XIV ${ }^{\mathrm{e}}$ siècle $^{66}$. Quant à Charles le Téméraire, le dernier duc de Valois-Bourgogne, il reprit à la suite de ses prédécesseurs cette politique d'ostentation avec l'aide de son orfèvre, Gérard Loyet. Des aquarelles représentant certains de ses joyaux, aujourd'hui perdus, permettent d'ailleurs d'avantage de se figurer l'éclat et la richesse des costumes et bijoux bourguignons ${ }^{67}$.

${ }^{65}$ Cette expression désigne les quatre fils du roi Jean le Bon : Charles V de France, Philippe le Hardi, duc de Bourgogne, Louis d'Anjou et Jean de Berry.

${ }^{66}$ L'inventaire de Philippe le Bon, duc de Bourgogne, fut réalisé en 1420 pour Philippe le Bon, mais répertorie des objets ayant en majorité appartenu à son père, Jean sans Peur.

${ }^{67}$ Hugo VAN DER Velden, The Donor's Image..., op. cit., p. 144-145. 